\title{
ODD DIMENSIONAL MANIFOLDS WITH REGULAR CONJUGATE LOCUS
}

\author{
F. MERCURI
}

\begin{abstract}
We show that all odd dimensional manifolds, for which the first conjugate locus, with respect to some point, is regular, are homeomorphic to a sphere.
\end{abstract}

In [2] Warner classifies, almost completely, simply connected, complete Riemannian manifolds $M^{n}$ for which there exists a point $p \in M^{n}$ whose first conjugate locus is regular. Almost completely means that he needs one of the following two conditions:

(1) The first conjugate point with respect to $p$ in any direction has order $k \geqq 2$.

(2) All the first conjugate points with respect to $p$ lie at the same distance from $p$.

The purpose of this note is to observe that in the odd dimensional case neither assumption is necessary.

Proposition. Let $M^{n}$ be a complete simply connected Riemannian manifold, $n$ odd, and suppose there exists $p \in M^{n}$ such that the first conjugate point with respect to $p$ exists in any direction and has constant order $k$. Then $k=n-1$ and $M^{n}$ is homeomorphic to $S^{n}$.

Proof. If we show that $k>1$ then Warner's condition (1) is satisfied and $n$ odd implies $k=n-1$ and $M^{n}$ homeomorphic to $S^{n}$. (See [2].)

Suppose $k=1$. Let $C(p)$ denote the first conjugate locus with respect to $p$. Then $C(p)$ is a smooth closed submanifold of $M_{p}$ diffeomorphic to an even $(n-1)$-dimensional sphere and transverse to the lines through the origin in $M_{p}$ (see [1]). For $x \in C(p), \operatorname{Ker}\left(d \exp _{p}\right)_{x}$ is orthogonal to the line $\{t x \mid t \in \boldsymbol{R}\}$ by Gauss' lemma and therefore has a nontrivial projection on the tangent space $C(p)_{x}$. In this way we can define a 1-dimensional distribution on $C(p)$, i.e., a 1-dimensional tangent line bundle that is trivial, since $C(p)$ is diffeomorphic to a sphere, and therefore define a nowhere zero vector field. But this is impossible since $n-1$ is even.

Received by the editors August 17, 1972.

AMS (MOS) subject classifications (1970). Primary 53C20.

Key words and phrases. Riemannian manifold, regular conjugate locus.

(C) American Mathematical Society 1973 
REMARK. It is easily seen that a manifold for which there exists a point whose first conjugate locus has order $n-1$ is diffeomorphic to the union of two disks. Conversely, if $M^{n}=D^{n} \cup_{g} D^{n}, g: \partial D^{n} \rightarrow \partial D^{n}$ being a diffeomorphism, it is always possible to put a metric on $M^{n}$ such that there exists a point whose first conjugate locus has order $n-1$ [2]. Therefore no better result is possible under these hypotheses.

\section{REFERENCES}

1. F. Warner, The conjugate locus of a Riemannian manifold, Amer. J. Math. 87 (1965), 575-604. MR 34 \#8344.

2. - Conjugate loci of constant order, Ann. of Math. (2) 86 (1967), 192-212. MR 35 \#4851.

Department of Mathematics, University of Chicago, Chicago, Illinois 60637 